\title{
Evaluating the effects of crystallinity in new biocompatible polyester nanocarriers on drug release behavior
}

This article was published in the following Dove Press journal:

International Journal of Nanomedicine

23 November 2011

Number of times this article has been viewed

\author{
Vassilios Karavelidis ${ }^{1,2}$ \\ Evangelos Karavas ${ }^{2}$ \\ Dimitrios Giliopoulos' \\ Sofia Papadimitriou' \\ Dimitrios Bikiaris' \\ 'Laboratory of Polymer Chemistry \\ and Technology, Chemistry \\ Department, Aristotle University of \\ Thessaloniki, Thessaloniki, \\ 2Pharmathen SA, Pharmaceutical \\ Industry, Pallini Attikis, Attiki, Greece
}

Correspondence: Dimitrios Bikiaris Laboratory of Polymer Chemistry and Technology, Chemistry Department, Aristotle University of Thessaloniki,

54I 24 Thessaloniki, Greece

$\mathrm{Tel}+302310997812$

Fax +30231099 7769

Email dbic@chem.auth.gr

\begin{abstract}
Four new polyesters based on 1,3-propanediol and different aliphatic dicarboxylic acids were used to prepare ropinirole HCl-loaded nanoparticles. The novelty of this study lies in the use of polyesters with similar melting points but different degrees of crystallinity, varying from $29.8 \%$ to $67.5 \%$, as drug nanocarriers. Based on their toxicity to human umbilical vein endothelial cells, these aliphatic polyesters were found to have cytotoxicity similar to that of polylactic acid and so may be considered as prominent drug nanocarriers. Drug encapsulation in polyesters was performed via an emulsification/solvent evaporation method. The mean particle size of drug-loaded nanoparticles was $164-228 \mathrm{~nm}$, and the drug loading content was $16 \%-23 \%$. Wide angle X-ray diffraction patterns showed that ropinirole $\mathrm{HCl}$ existed in an amorphous state within the nanoparticle polymer matrices. Drug release diagrams revealed a burst effect for ropinirole $\mathrm{HCl}$ in the first 6 hours, probably due to release of drug located on the nanoparticle surface, followed by slower release. The degree of crystallinity of the host polymer matrix seemed to be an important parameter, because higher drug release rates were observed in polyesters with a low degree of crystallinity.
\end{abstract}

Keywords: biocompatible polyesters, nanoparticles, ropinirole, release, crystallinity

\section{Introduction}

Today's pharmaceutical formulation demands require that drug delivery systems are precise in their control of drug distribution and, preferably, respond directly to the local pathological environment in order to achieve a dynamic and beneficial interaction with the host pathology or physiology. ${ }^{1,2}$ The development of new biodegradable and biocompatible polymers capable of releasing physically incorporated therapeutic agents with well defined kinetics is a subject that has been receiving an increasing amount of attention. ${ }^{3,4}$ Aliphatic polyesters, due to their favorable features of biodegradability and biocompatibility, are one of the most important classes of synthetic biodegradable polymers and are nowadays available commercially in a variety of types. ${ }^{4,5}$ These polyesters are appropriate for medical and biomedical applications, including drug delivery systems, when prepared as drug-loaded nanoparticles or solid dispersions.

A significant effort has been made to develop appropriate nanocarriers for drug delivery because nanotechnology offers a suitable means of delivering small molecular weight drugs, as well as macromolecules, such as proteins, peptides or genes, by either localized or targeted delivery to the tissue of interest. ${ }^{6-8}$ Nanotechnology focuses on incorporating therapeutic agents into biocompatible nanocomposites, such as nanoparticles, nanocapsules, micellar systems, and conjugates, using different materials 
and techniques. ${ }^{910}$ These systems in general can be used to provide targeted delivery of drugs, to improve oral bioavailability, to sustain drug effects in target tissue, to solubilize drugs for intravascular delivery, and to improve the stability of therapeutic agents against enzymatic degradation (nucleases and proteases), especially of proteins, peptides, and nucleic acids. ${ }^{11-13}$ Furthermore, targeted nanocarriers reduce drug toxicity, provide more efficient drug distribution and overcome resistance offered by the physiological barriers in the body because efficient delivery of drug to various parts of the body is directly affected by particle size. Nanocarriers also hold promise for delivery of biotech drugs to the anatomic extremities of the body. ${ }^{14}$

However, nanocarriers have some limitations. They are difficult to handle, store, and administer because of their susceptibility to aggregation. They are also unsuitable for less potent drugs, and can gain access to unintended environments with harmful consequences. ${ }^{14}$ Furthermore, release rates from some polymer nanoparticles are very low.

The determination of drug release behavior from polymer nanoparticles has been reported by many research groups to be a rather complicated process. ${ }^{15}$ Drug release can be affected by many factors, including polymer degradation, molecular weight, the thermal properties of the polymer, as well as the binding affinity between the drug and the polymer matrix, the capability of the polymer to incorporate a high amount of drug, size of the nanoparticles, and the hydrophilicity or hydrophobicity of the drug. ${ }^{16-18}$

One of the major questions that arises for these kinds of polyesters when used as drug nanocarriers is whether crystallinity affects the release profile of the model drug. The novelty of this study lies upon the investigation of polyesters that have similar melting points but different degrees of crystallinity. In our previous work, we studied polyesters with similar crystallinity but different melting points varying from $46.7^{\circ} \mathrm{C}$ to $166.4^{\circ} \mathrm{C}$ and their effects on nanoparticle characteristics. In the present study, we synthesized polyesters of different crystallinity but with almost the same melting point in an attempt to study how crystallinity affects the characteristic release profile of these nanocarriers, excluding the parameter of melting point. To our knowledge, no other research team until now has referred to or performed any studies related to the drug release rate in terms of the degree of crystallinity in a series of aliphatic polyester nanocarriers with similar melting points.

Taking into consideration all the above factors, the aim of the present study was to evaluate how the degree of crystallinity of different aliphatic polyesters affects the release profile of ropinirole $\mathrm{HCl}$, a model hydrophilic drug, from loaded nanoparticles. Four aliphatic polyesters, ie, poly(propylene azelate) (PPAz), poly(propylene pimalate) (PPPim), poly(propylene glutarate) (PPGlu), and poly(propylene adipate) (PPAd), with similar melting points and different crystallinity were synthesized, characterized, and used as drug nanocarriers for the encapsulation of ropinirole $\mathrm{HCl}$. Ropinirole $\mathrm{HCl}$ (4-[2-(dipropylamino)ethyl]1,3-dihydro-2H-indol-2-one) is a nonergoline dopamine $\mathrm{D}_{2}$-receptor agonist with antiparkinsonian properties.

\section{Materials and methods Materials}

Adipic acid (99\%), glutaric acid (99\%), pimelic acid (99\%), and azelaic acid (99\%) were purchased from Aldrich Chemical Company (St Louis, MO). 1,3-propanediol (CAS 504-63-2, purity > 99.7\%) was kindly supplied by Du Pont de Nemours and Company (Pembroke Pines, FL). Analytical grade tetrabutyl titanate was purchased from Aldrich and used as a catalyst. Polyphosphoric acid, used as a heat stabilizer, was supplied by Fluka (Milwaukee, WI). Ropinirole $\mathrm{HCl}$ was purchased from Ragactives (Valladolid, Spain) as a white to pale greenish-yellow powder with a melting range of $241^{\circ} \mathrm{C}-245^{\circ} \mathrm{C}$, a solubility of $133 \mathrm{mg} / \mathrm{mL}$ in water, and a molecular weight of 296.84 (260.38 as the free base). All other materials and solvents used were of analytical grade.

\section{Synthesis of polyesters}

Synthesis of aliphatic polyesters was performed following the two-stage melt polycondensation method (esterification and polycondensation) in a glass batch reactor. ${ }^{19}$ In brief, appropriate amounts of diacid and 1,3-propanediol in a molar ratio of 1:1.1 and the catalyst tetrabutyl titanate $\left(3 \times 10^{-4} \mathrm{~mol}\right.$ tetrabutyl titanate/mol diacid) were put into the reaction tube of a polycondensation apparatus. The apparatus with the reagents was evacuated several times and filled with argon in order to remove all oxygen. The reaction mixture was heated to $190^{\circ} \mathrm{C}$ in an argon atmosphere and stirred at a constant speed (500 rpm). This first step (esterification) was considered to be complete after collection of almost all the theoretical amount of $\mathrm{H}_{2} \mathrm{O}$, which was removed from the reaction mixture by distillation and collected in a graduated cylinder. In the second polycondensation step, polyphosphoric acid was added $\left(5 \times 10^{-4} \mathrm{~mol}\right.$ polyphosphoric acid $/ \mathrm{mol}$ diacid) and a vacuum (5.0 Pa) was applied slowly over about 30 minutes, to avoid excessive foaming and to minimize oligomer sublimation. The temperature during this time was 
slowly increased to $230^{\circ} \mathrm{C}$, and the stirring speed was also increased to $720 \mathrm{rpm}$. Polycondensation continued for about 60 minutes for all prepared polyesters. After the end of the polycondensation reaction, the polyesters were easily removed, milled, and washed with methanol. Four polyesters were prepared according to this procedure, ie, PPAd, PPGlu, PPPim, and PPAz.

\section{Polymer characterization} Intrinsic viscosity measurement

Intrinsic viscosity measurements were performed on the isolated polymers using an Ubbelohde capillary viscometer at $25^{\circ} \mathrm{C}$ in chloroform at a solution concentration of $1 \mathrm{wt} \%$.

\section{Gel permeation chromatography}

Gel permeation chromatography analysis was performed using a Waters $150 \mathrm{C}$ gel permeation chromatography column equipped with a differential refractometer as the detector and three Ultrastyragel $(103,104,105 \AA)$ columns in series. $\mathrm{CHCl}_{3}$ was used as the eluent $(1 \mathrm{~mL} /$ minute $)$ and the measurements were performed at $35^{\circ} \mathrm{C}$. Calibration was performed using polystyrene standards with a narrow molecular weight distribution.

\section{Differential scanning calorimetry}

Differential scanning calorimetry was performed on a Perkin-Elmer Pyris Diamond differential scanning calorimeter calibrated with high purity standards. Functioning of the system at low temperatures (down to $-65^{\circ} \mathrm{C}$ ) was achieved using a Perkin Elmer Intracooler 2P cooling accessory. Samples weighing $5 \pm 0.1 \mathrm{mg}$ were sealed in aluminum pans and scanned using the instrument in a nitrogen atmosphere. A cyclic scanning procedure was followed to record the thermal behavior of each polyester. The procedure involved: heating from $0^{\circ} \mathrm{C}$ to $40^{\circ} \mathrm{C}$ above the melting point of each sample at a heating rate of $20^{\circ} \mathrm{C} /$ minute and holding at this temperature for 2 minutes in order to remove any thermal history of the sample; rapid cooling to $-65^{\circ} \mathrm{C}$ and equilibration; reheating at a heating rate $2.5^{\circ} \mathrm{C} /$ minute from $-65^{\circ} \mathrm{C}$ to $40^{\circ} \mathrm{C}$ above the melting temperature and holding for 2 minutes; and final cooling at a rate of $10^{\circ} \mathrm{C} /$ minute down to $-50^{\circ} \mathrm{C}$.

\section{Wide angle X-ray diffraction}

$\mathrm{X}$-ray diffraction measurements were performed using an automated powder diffractometer Rigaku Mini Flex II with Bragg-Brentano geometry $(\theta-2 \theta)$, using $\mathrm{CuK}_{\alpha}$ radiation $(\lambda=0.154 \mathrm{~nm})$ in the angle $2 \theta$ range of 5-60 degrees.

\section{Cytotoxicity study of prepared polyesters}

Human umbilical vein endothelial cells were grown routinely in RPMI-1640 medium supplemented with 15\% fetal bovine serum, $15 \mathrm{mg}$ endothelial cell growth supplement, penicillin $100 \mathrm{U} / \mathrm{mL}$, streptomycin $100 \mu \mathrm{g} / \mathrm{mL}$, gentamicin $50 \mu \mathrm{g} / \mathrm{mL}$, and amphotericin B $2.5 \mu \mathrm{g} / \mathrm{mL}$. Cultures were maintained at $37^{\circ} \mathrm{C}, 5 \% \mathrm{CO}_{2}$, and $100 \%$ humidity.

The cytotoxicity of the aliphatic polyesters in comparison with biocompatible polylactic acid was evaluated by measuring the viability of human umbilical vein endothelial cells in the presence of different concentrations of the polymers. Cell viability was determined by the '3-(4,5-dimethylthiazol2-yl)-2,5-diphenyl tetrazolium bromide) (MTT) assay. Human umbilical vein endothelial cells were seeded in 24-well plates at a density of 30,000 cells per well in $500 \mu \mathrm{L}$ cell culture medium. Twenty-four hours after plating, different amounts of aliphatic polyesters in the form of nanoparticles (suspended in culture medium) were added in the wells. After 24 hours of incubation at $37^{\circ} \mathrm{C}, 50 \mu \mathrm{L}$ of MTT solution ( $5 \mathrm{mg} / \mathrm{mL}$ in phosphate-buffered solution $\mathrm{pH} 7.4$ ) was added into each well and the plates were incubated at $37^{\circ} \mathrm{C}$ for 2 hours. The medium was withdrawn and $200 \mu \mathrm{L}$ of acidified isopropanol $(0.33 \mathrm{~mL} \mathrm{HCl}$ in $100 \mathrm{~mL}$ isopropanol) was added to each well and agitated thoroughly to dissolve the crystals formed. The solution was transferred to 96-well plates and immediately read on a microplate reader (Biorad, Hercules, CA) at a wavelength of $490 \mathrm{~nm}$. The experiments were performed in triplicate. Biocompatibility of the polymers was expressed as percent cell viability, which was calculated from the ratio of the number of cells treated with the nanoparticles to that of nontreated cells (control).

\section{Preparation of ropinirole $\mathrm{HCl}$-loaded polyester nanoparticles}

Ropinirole $\mathrm{HCl}$ nanoencapsulation in polyester matrices was carried out using a water-oil-water (w/o/w) emulsification/ solvent evaporation technique. In brief, $50 \mathrm{mg}$ of the polyester was dissolved in $2 \mathrm{~mL}$ of dichloromethane and mixed with $0.5 \mathrm{~mL}$ of $10 \mathrm{mg} / \mathrm{mL}$ ropinirole $\mathrm{HCl}$ aqueous stock solution. The mixture was sonicated for 1 minute. Then, $5.5 \mathrm{~mL}$ of $12 \mathrm{mM}$ sodium cholate aqueous solution was added and the new mixture was sonicated for 1 minute. Sodium cholate was added to prevent drug particle aggregation during solvent evaporation. As a result, the drug-loaded polymer was dispersed in the form of nanoparticles. The emulsion formed was gently stirred until evaporation of the organic solvent was complete. The nanoparticles were purified by centrifugation (9500 rpm for 20 minutes). The samples were 
reconstituted with deionized water. Polymer aggregates were removed by filtering the suspension through a $1.2 \mu \mathrm{m}$ pore size microfilter.

\section{Characterization of drug-loaded nanoparticles}

\section{Nanoparticle yield, drug loading, and entrapment efficiency}

For determination of drug loading content, $3 \mathrm{mg}$ of the nanoparticles was dissolved in $50 \mu \mathrm{L}$ dichloromethane and diluted to $1 \mathrm{~mL}$ using phosphate buffer ( $\mathrm{pH} 3 / \mathrm{ACN}$ 65/35 v/v solution). A clear solution was obtained for high-pressure liquid chromatographic analysis. Quantitative analysis of the synthesized nanoparticles was carried out using a Shimadzu high-pressure liquid chromatographic column (model LC-20 AD). The column used was a Hypersil BDS, $5 \mu \mathrm{m}$, $200 \times 4.6 \mathrm{~mm}$ with a column temperature of $30^{\circ} \mathrm{C}$. The mobile phase consisted of phosphate buffer at a flow rate of $1 \mathrm{~mL} /$ minute. Concentration determination was performed using high-pressure liquid chromatography-ultraviolet apparatus at $250 \mathrm{~nm}$, and was based on a calibration curve previously created by diluting a stock aqueous solution of $1 \mathrm{mg} / \mathrm{mL}$ ropinirole $\mathrm{HCl}$ to concentrations of $80,40,20,10,5,1$, and $0.5 \mu \mathrm{g} / \mathrm{mL}$ using the same mobile phase mentioned above. Nanoparticle yield, drug loading, and drug entrapment efficiency were calculated from equations (1) to (3), respectively:

$$
\begin{aligned}
& \text { Nanoparticles yield }(\%) \\
& \quad=\frac{\text { Weight of nanoparticles }}{\text { Weight of polymer and drug fed initially }} \times 100 \\
& \text { Drug loading }(\%) \\
& \quad=\frac{\text { Weight of drug in nanoparticles }}{\text { Weight of nanoparticles }} \times 100 \\
& \text { Entrapment efficiency }(\%) \\
& \quad=\frac{\text { Weight of drug in nanoparticles }}{\text { Weight of drug fed initially }} \times 100
\end{aligned}
$$

\section{Morphology}

The morphology of the prepared nanoparticles was examined by scanning electron microscopy (JMS-840, JEOL Inc, Peabody, MA). The samples were coated with carbon black to avoid any charging under the electron beam. The operating conditions included an accelerating voltage of $20 \mathrm{kV}$, a probe current of $45 \mathrm{nA}$, and a counting time of 60 seconds.

\section{Particle size distribution}

The particle size distribution of the ropinirole $\mathrm{HCl}$ polyester nanoparticles was determined by dynamic light scattering using a Zetasizer (Nano ZS, ZEN3600, Malvern Instruments, Worcestershire, UK) operating with a $532 \mathrm{~nm}$ laser. A suitable amount of nanoparticles was dispersed in distilled water, creating a total concentration of $1 \%$, and this was kept at $37^{\circ} \mathrm{C}$ and agitation at $100 \mathrm{rpm}$. Particle size was measured at different time intervals after introduction of the sample into the dispersed medium. All measurements were performed in triplicate and the results are reported as the mean diameter \pm standard deviation.

\section{In vitro drug release studies}

The rate of ropinirole $\mathrm{HCl}$ release from the nanoparticles was measured in dissolution apparatus (DISTEK 2100B) equipped with an autosampler using the paddle (USP II) method. Each dissolution vessel was loaded with a quantity of nanoparticles corresponding to $2.5 \mathrm{mg}$ of ropinirole $\mathrm{HCl}$. The test was performed at $37^{\circ} \mathrm{C} \pm 1^{\circ} \mathrm{C}$ with a rotation speed of $100 \mathrm{rpm}$. The dissolution medium was $500 \mathrm{~mL}$ of phosphate buffer ( $\mathrm{pH}$ 7.4). At predetermined time intervals, samples of $5 \mathrm{~mL}$ were withdrawn from the dissolution medium, filtered through $45 \mu \mathrm{m}$ ultrahigh molecular weight polyethylene filters, and assayed using a high-pressure liquid chromatography-ultraviolet method for the drug at $250 \mathrm{~nm}$. An equal volume of fresh dissolution medium was transferred to the vessel after withdrawal of the sample. All measurements were performed in triplicate.

\section{Results and discussion Polymer characterization}

Synthesis and characterization of polyesters derived from the reaction of 1,3-propanediol and different dicarboxylic acids, as well as their biodegradability, have been discussed analytically in a previous study. ${ }^{20}$ Our polyester characterization results for intrinsic viscosity and molecular weight are presented in Table 1. In brief, both intrinsic viscosity and gel permeation chromatography measurements demonstrated high molecular weight values for the prepared polyesters. However, there were some differences in $M_{n}$ values, with PPAd and PPGlu having the lower $\mathrm{M}_{\mathrm{n}}$ of 14,000 Da and PPAz having a higher $M_{n}$ of 25,000 Da.

Polyesters exhibit variable thermal behavior according to the number of methylene groups in the repeating unit, as well as the number of monomer units. However, the polyesters selected for the present study had similar melting points in the range of $43.3^{\circ} \mathrm{C}-57.1^{\circ} \mathrm{C}$. After melting, the samples 
Table I Intrinsic viscosity [ $\eta$ ], average molecular weight $\left(M_{n}\right)$, and thermal properties of the prepared polyesters

\begin{tabular}{lllllll}
\hline & $\begin{array}{l}{[\eta]} \\
(\mathbf{d L} / \mathbf{g})\end{array}$ & $\begin{array}{l}\mathbf{M}_{\mathrm{n}} \\
\mathbf{( \mathbf { D a } )}\end{array}$ & $\begin{array}{l}\mathbf{T}_{\mathbf{m}} \\
\left({ }^{\circ} \mathbf{C}\right)\end{array}$ & $\begin{array}{l}\mathbf{T}_{\mathbf{g}} \\
\left({ }^{\circ} \mathbf{C}\right)\end{array}$ & $\begin{array}{l}\mathbf{T}_{\mathrm{cc}} \\
\left({ }^{\circ} \mathbf{C}\right)\end{array}$ & $\begin{array}{l}\Delta \mathbf{H}_{\mathbf{m}} \\
\mathbf{( J / g})\end{array}$ \\
\hline PPAd & 0.57 & 14,000 & 43.3 & -58.8 & -1.1 & 48.1 \\
PPGlu & 0.58 & 14,000 & 45.9 & -45.0 & - & 46.0 \\
PPPim & 0.70 & 19,000 & 44.9 & -56.9 & -29.6 & 53.7 \\
PPAz & 0.84 & 25,000 & 57.1 & -46.7 & - & 65.7 \\
\hline
\end{tabular}

Abbreviations: PPAz, poly(propylene azelate); PPPim, poly(propylene pimalate) PPGlu, poly(propylene glutarate); PPAd, poly(propylene adipate).

were rapidly cooled in the differential scanning calorimetry instrument down to $-65^{\circ} \mathrm{C}$, and a second scan was performed in the quenched samples to record the glass transition and cold crystallization of the amorphous polyesters. PPAd has the lowest glass transition temperature at $-58.8^{\circ} \mathrm{C}$ while PPGlu has the highest at $-45.0^{\circ} \mathrm{C}$. However, it can be said that these aliphatic polyesters show increased chain flexibility since their glass transition temperatures are very low. PPAz and PPPim also had very low glass transition temperatures at $-46.7^{\circ} \mathrm{C}$ and $-56.9^{\circ} \mathrm{C}$, respectively. A slow heating rate of $2.5^{\circ} \mathrm{C} /$ minute was used in the second scan to record the cold crystallization of the quenched polyesters. However, as can be seen, PPGlu and PPAz could not be crystallized during heating, and only PPAd and PPPim gave a cold crystallization temperature. The different crystallization rates may also

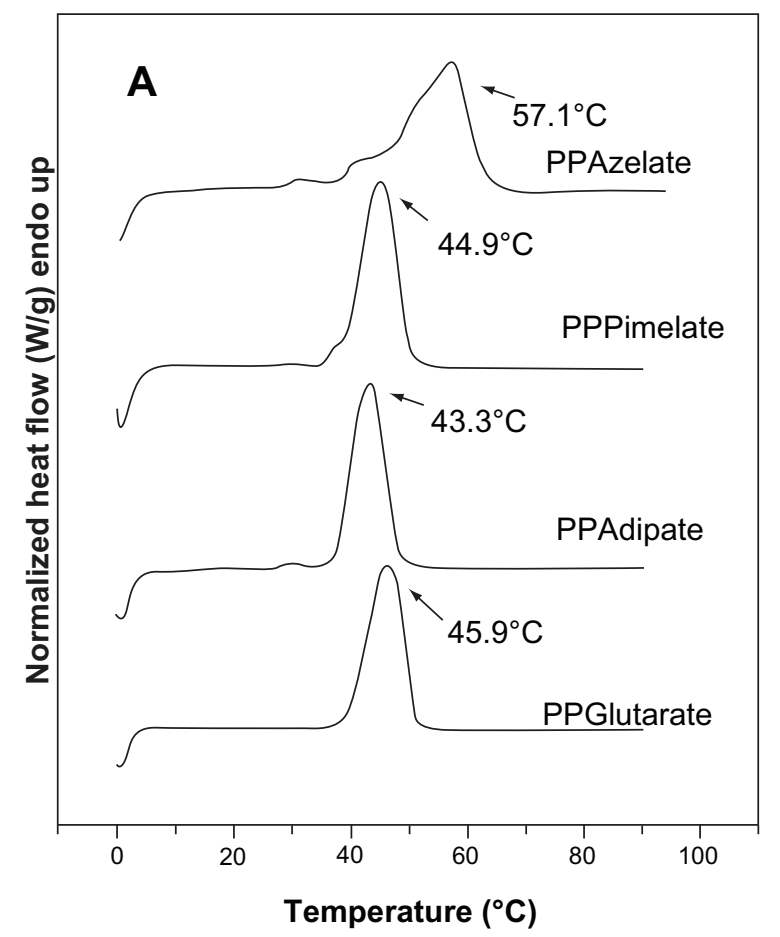

affect the degree of crystallinity of the polyesters. The respective heating scans (first scan and after quenching) are shown in Figure 1.

It was demonstrated in a previous study that the melting points and glass transition temperatures of polyesters may play an important role in their drug release characteristics. ${ }^{21}$ Polyesters with a high melting point give a slower drug release while those having melting points close to that of the human body have higher drug release rates. Similar behavior was observed for the glass transition temperatures of the polyesters. With these results in mind, polyesters with very similar melting points and glass transition temperatures were selected as candidate nanocarriers for the present study. As shown in Table 1, there is no great variation in melting points and glass transition temperatures for the selected aliphatic polyesters which would affect evaluation of the effect of other physical characteristics and, in particular, the effect of degree of crystallinity on drug release from these polymeric nanoparticles. From the heat of fusion values measured in the heating traces of the samples as received, which crystallized during 2 months of storage at room temperature before scanning, the degree of crystallinity values can be calculated. PPAz and PPPim show higher heat of fusion values compared with PPGlu and PPAd in Table 1. In order to calculate the degree of crystallinity for each polyester, the heat of fusion of the

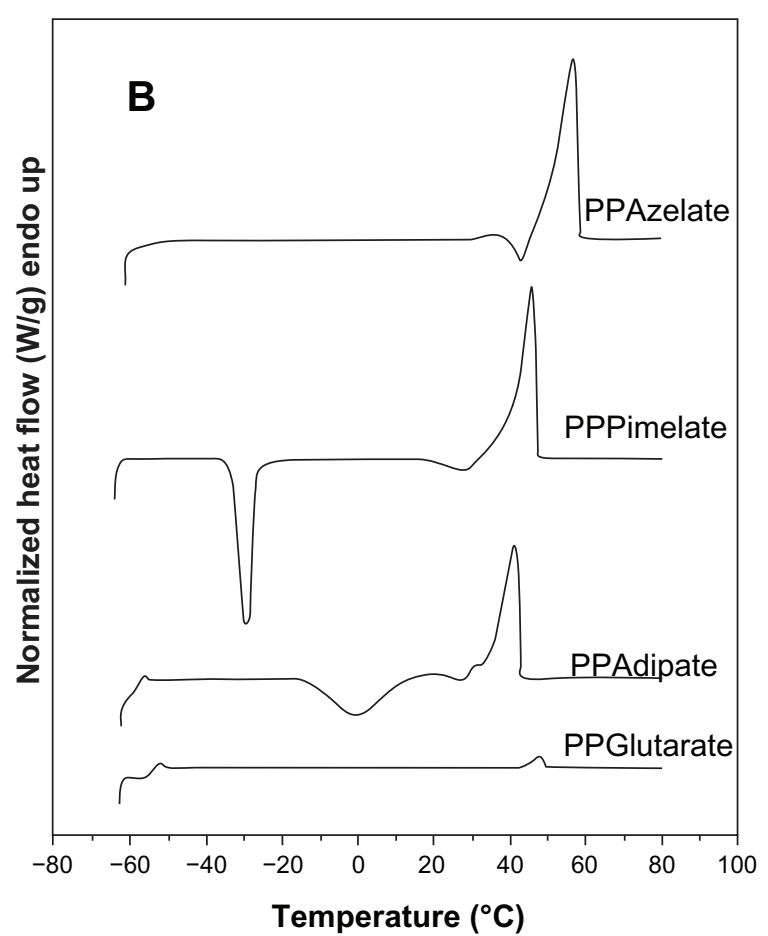

Figure I Differential scanning calorimetry thermograms of the studied aliphatic polyesters. (A) First scan of polyesters as received and kept at room temperature for 2 months, and (B) a second scan of polyesters after quenching.

Abbreviations: PPAz, poly(propylene azelate); PPPim, poly(propylene pimalate); PPGlu, poly(propylene glutarate); PPAd, poly(propylene adipate). 
perfect crystal (100\% crystal material) should be known, but is not. For this reason, the degree of crystallinity of all the polyesters studied was calculated using wide angle X-ray diffraction patterns (Figure 2).

Although there are some differences in the intensities of the peaks, the wide angle X-ray diffraction patterns are similar for PPAz, PPPim, and PPGlu, because the characteristic diffractions peaks appear at about the same diffraction angles of $2 \theta$. The unit cells of the polymers in this group are probably similar, and some progressive changes in the dimensions occur with increasing length of the repeating unit of dicarboxylic acid, given that propylene glycol is the same in all polyesters. However, for PPAd, which has the highest degree of crystallinity, a double peak is obtained at $2 \theta 19.9$ and 20.8, which is not recorded for the other polyesters. The crystallinity values were calculated from wide angle X-ray diffraction patterns using the relative areas under the crystalline peaks $\left(A_{c}\right)$ and the amorphous background $\left(A_{a}\right)$ using equation 3 , according to $\mathrm{Lu}$ and Hay, ${ }^{22}$ and are summarized in Table 2.

$$
X_{c}=\left(1+\frac{A_{a m}}{A_{c}}\right)^{-1}
$$

Crystallinity values were calculated using a special program on the diffractometer and are summarized in Table 2.
As can be seen, PPAd has the highest degree of crystallinity at $67.5 \%$, while PPAz has the lowest at 29.8\%. PPPim and PPGlu give intermediate values of $43.9 \%$ and $55.4 \%$, respectively. The difference between the aliphatic polyesters in this respect is considerable, and is expected to play the most important role in drug release.

\section{In vitro cytotoxicity of aliphatic polyesters}

Aliphatic polyesters such as polycaprolactone and polylactide are extensively used as drug carriers because they are biocompatible materials. The polyesters used in the present study are aliphatic and similar to polycaprolactone and polylactide. However, their in vitro biocompatibility needs to be evaluated in order for these compounds to be considered as potential drug nanocarriers. Use of similar aliphatic polyesters derived from 1,3 propanediol and carboxylic acids as nanocarriers has been previously studied and reported. ${ }^{23-25}$

These polyesters have been used previously for the preparation of a solid dispersion by melt mixing, while some of their copolymers have been effective for nanoencapsulation and sustained release of nimodipine. ${ }^{25}$ Figure 3 demonstrates that all the polyesters studied had low toxicity in human umbilical vein endothelial cells, with appreciable

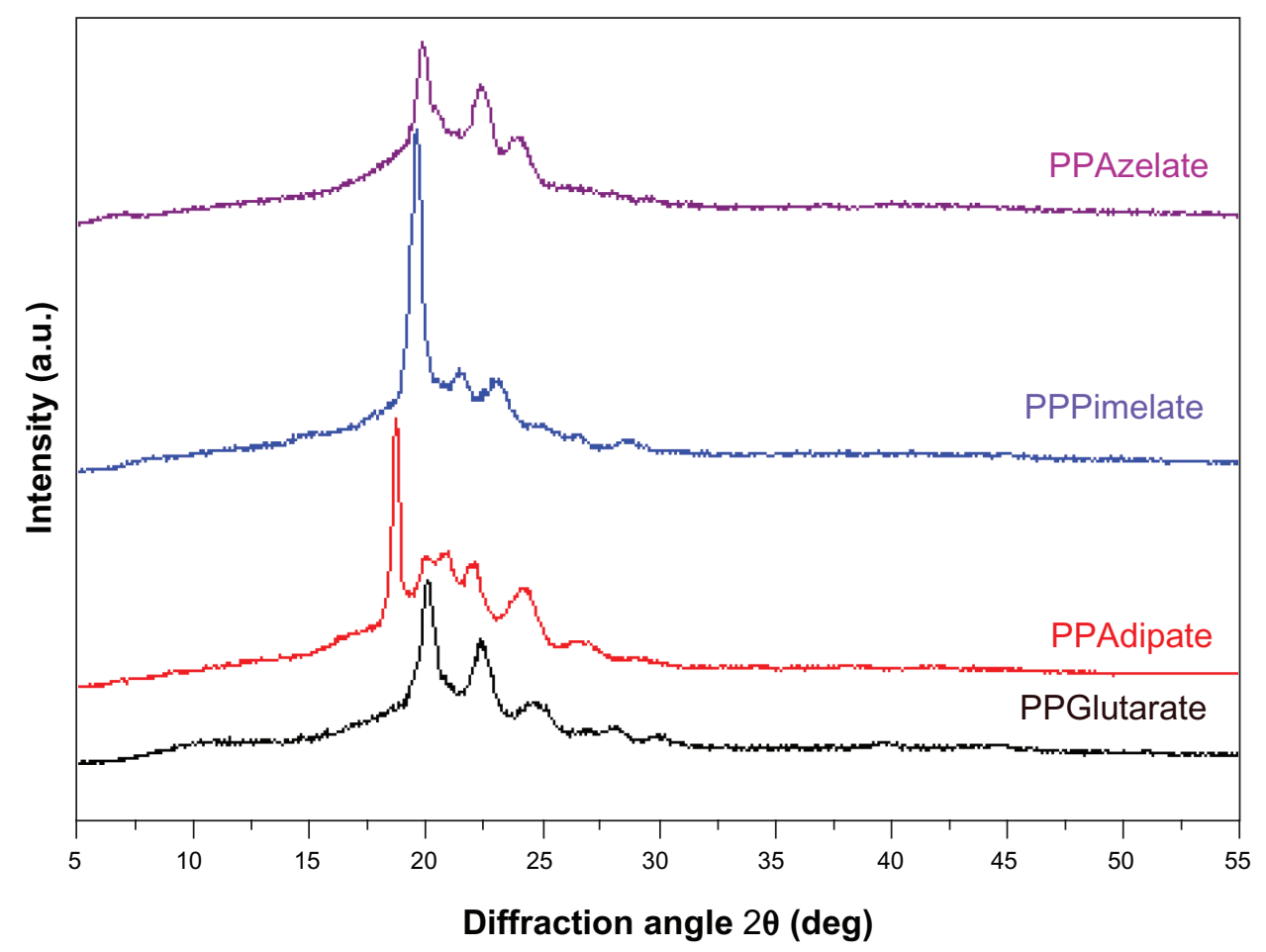

Figure 2 Wide angle X-ray diffraction patterns of the studied aliphatic polyesters.

Abbreviations: PPAz, poly(propylene azelate); PPPim, poly(propylene pimalate); PPGlu, poly(propylene glutarate); PPAd, poly(propylene adipate). 
Table 2 Diffraction angles $2 \theta$ for poly(propylene alkanedicarboxylate) crystals

\begin{tabular}{|c|c|c|c|c|c|c|c|c|}
\hline \multirow{2}{*}{$\begin{array}{l}\text { Polyester } \\
\text { PPAz }\end{array}$} & \multicolumn{7}{|c|}{ Diffraction angle $2 \theta$ (degrees) } & \multirow{2}{*}{$\begin{array}{l}\text { Degree of crystallinity (\%) } \\
29.8\end{array}$} \\
\hline & & 19.90 & 22.36 & 23.98 & & & & \\
\hline PPPim & & 19.64 & 21.45 & 23.09 & 25.06 & 26.43 & 28.67 & 43.9 \\
\hline PPGlu & & 20.04 & 24.70 & 28.07 & 30.00 & 39.62 & 44.72 & 55.4 \\
\hline PPAd & |8.7| & 19.90 & 20.80 & 22.09 & 24.14 & 26.65 & & 67.5 \\
\hline
\end{tabular}

Abbreviations: PPAz, poly(propylene azelate); PPPim, poly(propylene pimalate); PPGlu, poly(propylene glutarate); PPAd, poly(propylene adipate).

cytotoxicity (more than $20 \%$ reduction of cell viability) being observed only after exposing the cells to high nanoparticle concentrations, ie, higher than $800 \mu \mathrm{g} / \mathrm{mL}$. Based on their toxicity to human umbilical vein endothelial cells, the biocompatibility of polyesters was comparable with that of polylactide, which is a polymer of high biocompatibility and has a wide variety of biomedical applications. ${ }^{26}$ The results of our present study indicate that these polyesters may be considered as novel nanocarriers.

\section{Nanoparticle characterization}

The polyesters prepared were used for drug encapsulation in the form of nanoparticles. The main nanoparticle characteristics, such as nanoparticle yield, drug loading, and encapsulation efficiency, as well as nanoparticle size, are presented in Table 3. Drug loading content for all the polyesters varied between $16 \%$ and $23 \%$, and entrapment efficiency was relatively high. PPGlu had the highest entrapment efficiency (61\%) while PPAd had the lowest $(49 \%)$. Drug loading content and entrapment efficiency values were quite satisfactory, considering that ropinirole $\mathrm{HCl}$ is a highly water-soluble drug and is rather difficult to encapsulate into these hydrophobic aliphatic polyesters. Similar data were also obtained in previous studies using an emulsification solvent evaporation method for drug encapsulation in aliphatic polyesters with a polymer matrix. ${ }^{21,25}$ Several factors may affect drug loading content and encapsulation efficiency, including affinity of the loaded drug for the preformed polymer, hydrophobicity of the polymer matrix, drug solubility in water, and drug-drug interaction, ie, its ability to self-aggregate. ${ }^{27,28}$ However, in the present study, the polyesters showed no trends with regard to drug loading and entrapment efficiency, so it may be stated that differences in degree of crystallinity may not play an important role in polyesters.

Nanoparticle size is an important parameter because it can affect drug release, physical stability, and uptake by cells. Nanoparticle yield and size distribution may be affected by a number of parameters, including stirring rate, type and amount of dispersing agent, viscosity of organic and aqueous

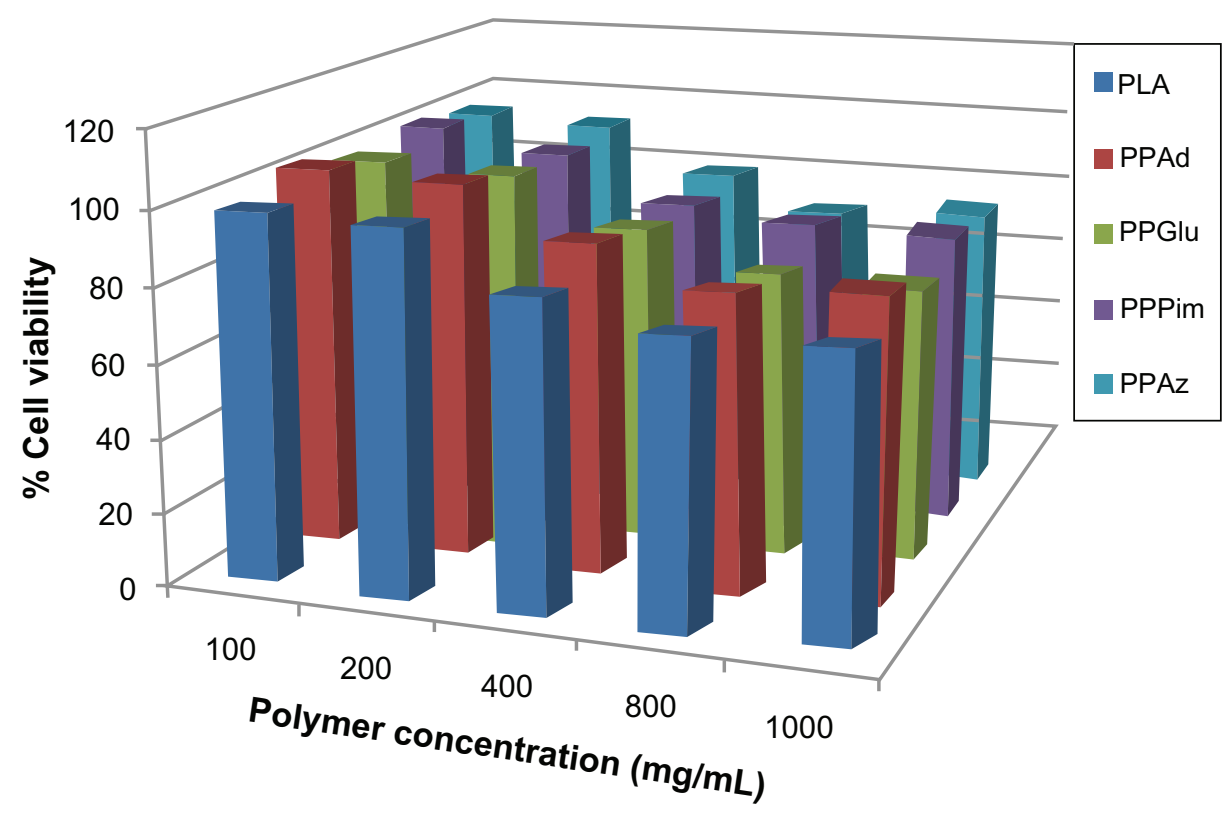

Figure 3 Human umbilical vein endothelial cell viability after incubation for 24 hours with different polymers and different polymer concentrations. Abbreviations: PPAz, poly(propylene azelate); PPPim, poly(propylene pimalate); PPGlu, poly(propylene glutarate); PPAd, poly(propylene adipate); PLA, polylactic acid. 
Table 3 Nanoparticle yield, drug loading content, and entrapment efficiency of the polyesters

\begin{tabular}{lllll}
\hline Polyester & $\begin{array}{l}\text { Nanoparticle } \\
\text { yield (\%) }\end{array}$ & $\begin{array}{l}\text { Drug loading } \\
\text { content (\%) }\end{array}$ & $\begin{array}{l}\text { Entrapment } \\
\text { efficiency (\%) }\end{array}$ & $\begin{array}{l}\text { Particle } \\
\text { size (nm) }\end{array}$ \\
\hline PPAz & $36 \pm 6$ & $19 \pm 4$ & $57 \pm 7$ & $228 \pm 14$ \\
PPPim & $28 \pm 10$ & $18 \pm 3$ & $52 \pm 5$ & $192 \pm 12$ \\
PPGlu & $30 \pm 12$ & $23 \pm 4$ & $61 \pm 7$ & $164 \pm 12$ \\
PPAd & $31 \pm 8$ & $16 \pm 3$ & $49 \pm 5$ & $175 \pm 15$ \\
\hline
\end{tabular}

Note: All measurements were performed in triplicate.

Abbreviations: PPAz, poly(propylene azelate); PPPim, poly(propylene pimalate); PPGlu, poly(propylene glutarate); PPAd, poly(propylene adipate).

phases, and temperature, ${ }^{29}$ as well as encapsulation efficiency and drug loading. The size (Table 3) and distribution (Figure 4) of the nanoparticles was measured by light scattering. It is obvious that all the nanoparticles in this study showed a unimodal size distribution. The mean nanoparticle diameter for the polyesters varied from a minimum of $164 \mathrm{~nm}$ for PPGlu to a maximum of $228 \mathrm{~nm}$ for PPAz. Similarly small particle sizes were also observed in a previous study using the same kind of aliphatic polyesters for encapsulation of ropinirole $\mathrm{HCl}^{21}$ Furthermore, as has been previously reported for copolymer of lactic acid/glycolic acid nanoparticles, an increase in molecular weight leads to an increase in nanoparticle size. ${ }^{30}$ This may explain the nanoparticle size for PPAz and PPPim, because both materials have a higher molecular weight (Table 1). However, it should be mentioned that the polydispersity of the nanoparticles was high in all cases, and so their mean particle size should not have a significant effect on drug release.

Scanning electron micrographs of samples of the nanoparticles are shown in Figure 5, and establish that the drugloaded nanoparticles have a discrete spherical shape with different sizes ranging from $150 \mathrm{~nm}$ to $250 \mathrm{~nm}$. These results are in accordance with results obtained from dynamic light scattering measurements, but with some small differences due to the different methods used. Similar results have been previously reported elsewhere. ${ }^{31}$

Wide angle X-ray diffraction was used to identify the physical state of the drug incorporated into the polymeric nanoparticles, and the patterns of pure ropinirole $\mathrm{HCl}$ and drug-loaded nanoparticles are presented in Figure 6. Crystalline ropinirole $\mathrm{HCl}$ showed a large number of sharp diffraction peaks, whereas these characteristic peaks were not detected for the drug-loaded nanoparticles, in which only the characteristic diffraction peaks of aliphatic polyesters were recorded. Thus, it can be stated that ropinirole $\mathrm{HCl}$ was dispersed in an amorphous state in all the semicrystalline polymer matrices. As can be seen, the patterns of the

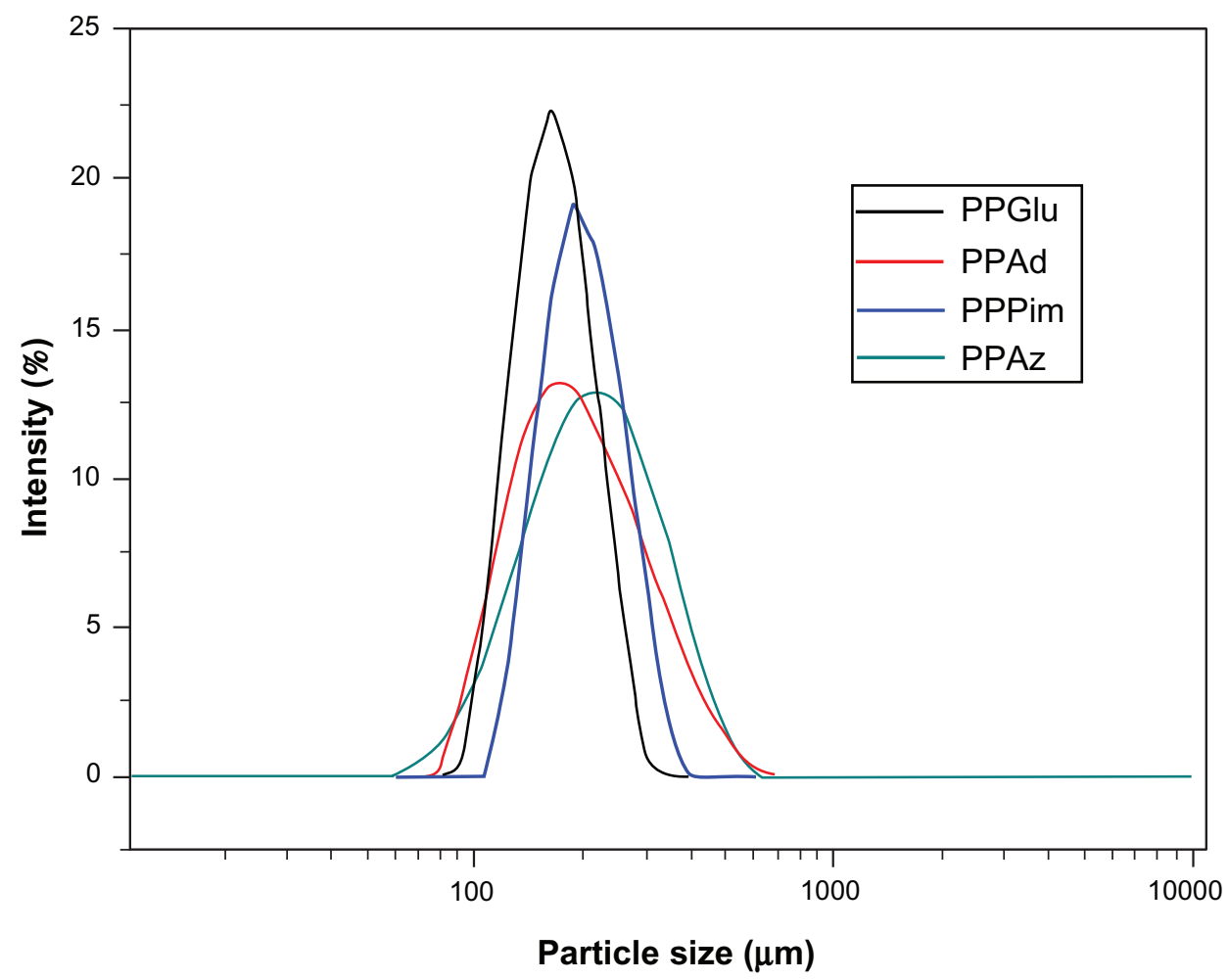

Figure 4 Particle size distribution of ropinirole $\mathrm{HCl}$-loaded nanoparticles.

Abbreviations: PPAz, poly(propylene azelate); PPPim, poly(propylene pimalate); PPGlu, poly(propylene glutarate); PPAd, poly(propylene adipate). 
A

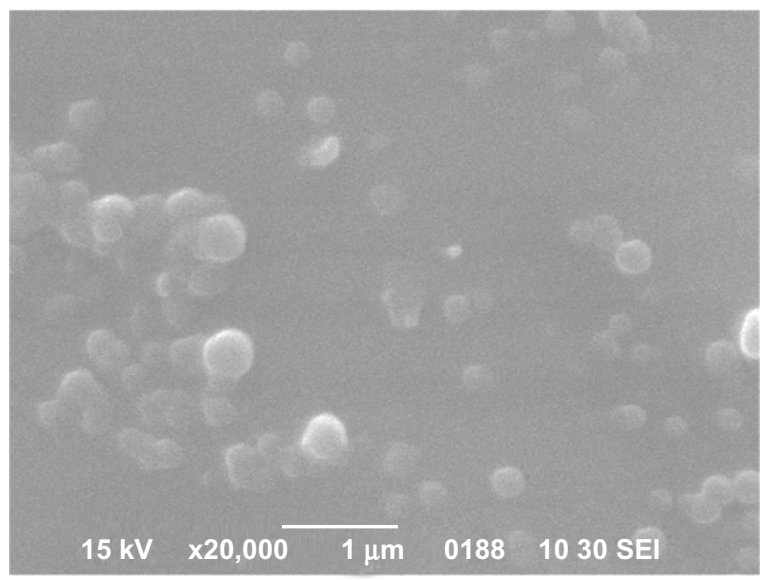

\section{B}

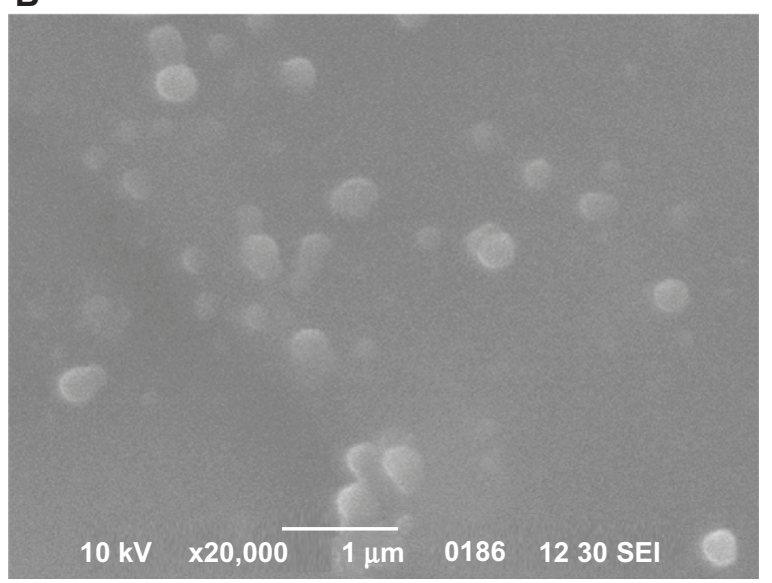

Figure 5 Scanning electron micrographs of ropinirole $\mathrm{HCl}$-loaded nanoparticles with the studied polyesters. (A) PPAz; (B) PPPim.

Abbreviations: PPAz, poly(propylene azelate); PPPim, poly(propylene pimalate).

nanoparticles are identical to that of the pure polyesters, indicating that nanoencapsulation of ropinirole $\mathrm{HCl}$ into polyester nanoparticles does change their crystalline form. The degree of crystallinity in the nanoparticles was calculated from these patterns. In the case of PPAz, crystallinity was about $29.5 \%, 42.6 \%$ in PPPim, $52.9 \%$ in PPGlu, and $62.6 \%$ in PPAd. These are very close to those reported for the pure aliphatic polyesters, but slightly smaller values were detected in all the nanoparticles. This is probably due to the wide angle X-ray diffraction patterns being recorded just after preparation of the nanoparticles and not enough time being available to reach a higher degree of crystallinity.

\section{In vitro drug release}

Aliphatic polyesters are appropriate vehicles for encapsulating all kinds of active pharmaceutical ingredients, whether hydrophilic or hydrophobic. It is possible to control drug release using an appropriate kind of aliphatic polyester. Furthermore, using the same polyester and changing its physical characteristics, such as its molecular weight, it is possible to modify drug release in order to achieve the desired drug release behavior. ${ }^{30} \mathrm{~A}$ lot of other parameters can also affect drug release from nanoparticles, such as the polymer degradation rate, the binding affinity between the polymer and the drug, the capability of the polymer to incorporate high amounts of drug, nanoparticle size, and drug hydrophilicity. ${ }^{16,32}$ In a previous study, release of ropinirole $\mathrm{HCl}$ from polyester nanoparticles with similar crystallinity and different melting points was examined, and it was verified that melting point plays a critical role in drug dissolution behavior. ${ }^{21}$ In fact, polyesters with melting points near human body temperature show enhanced drug release. In the present work, we studied how drug release is affected when aliphatic polyesters with similar melting points but different degrees of crystallinity were used as nanomatrices. Figure 7 shows the ropinirole $\mathrm{HCl}$ release profiles for the prepared nanoparticles. In all cases, a burst release was observed during the early stages of dissolution (up to 6-8 hours), followed by a phase of relatively slow drug release (until 24 hours). The main factor contributing to the fast release of ropinirole $\mathrm{HCl}$ from the nanoparticles into the aqueous dissolution medium is that ropinirole $\mathrm{HCl}$ is a hydrophilic drug with high solubility. It has been reported in the literature that most drug-loaded nanoparticle formulations show a biphasic release pattern in which an initial burst is followed by slow release rates from the internal phase of nanoparticles. ${ }^{33}$ In the present study, a characteristic burst effect was also detected, which appears probably due to the fraction of drug located on (or close to) the surface of the nanoparticles, ${ }^{33}$ as well as the high hydrophilicity and high solubility of ropinirole $\mathrm{HCl}$. Since the first w/o emulsion of the double emulsion method used here to encapsulate the hydrophilic ropinirole in the nanoparticles was not stabilized with a surfactant, migration of the hydrophilic drug to the external aqueous phase during the second emulsification step (and nanoparticle hardening process) might have occurred. As a result, most of the hydrophilic drug molecules that remained in the nanoparticles (ie, the drug fraction not lost to the external aqueous phase) may be located close to the surface, with only a small portion partitioned deep into the hydrophobic core, as has been reported previously. ${ }^{34}$ However, in this case, all nanoparticles should have similar behavior. Therefore, factors in addition to drug location are also responsible for such behavior. Of course, the high release rate of ropinirole $\mathrm{HCl}$ from nanoparticles at initial dissolution should be attributed to the high drug 


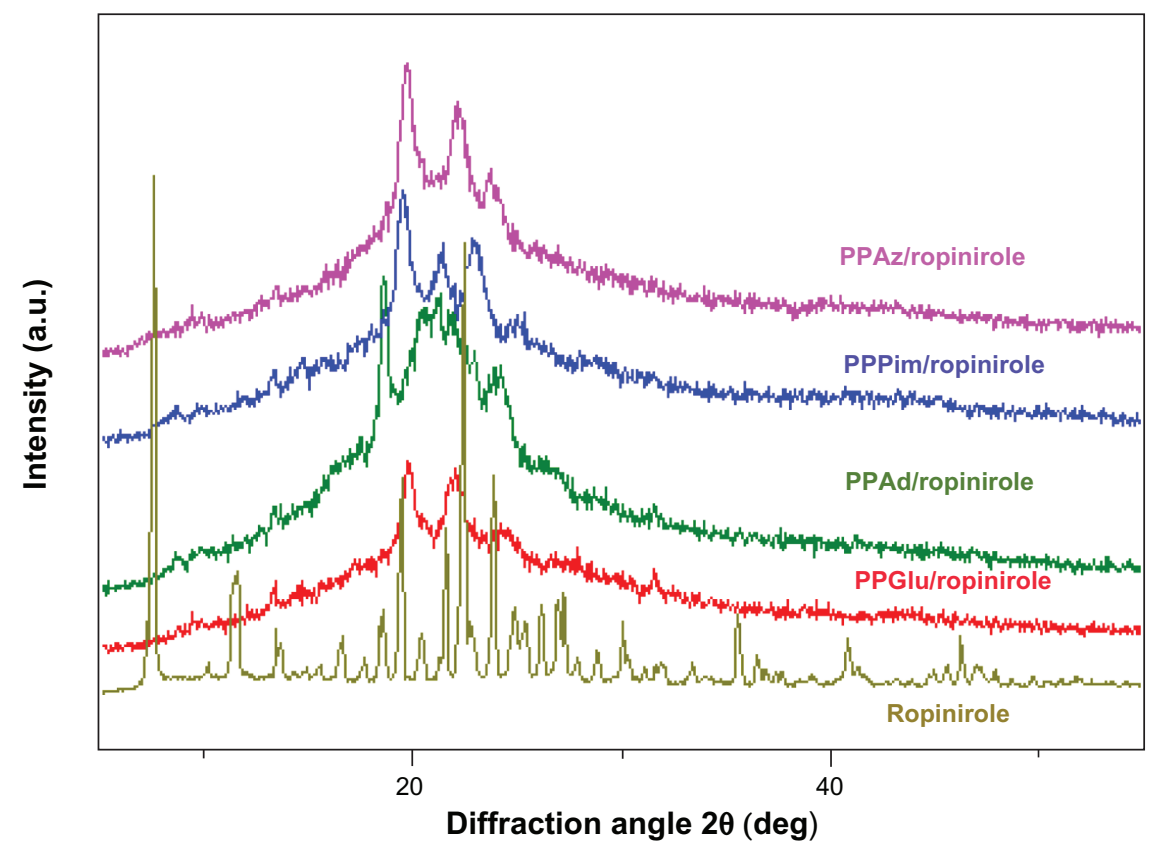

Figure 6 Wide angle $\mathrm{X}$-ray diffraction patterns for ropinirole $\mathrm{HCl}$ and ropinirole $\mathrm{HCl}$-loaded nanoparticles.

Abbreviations: PPAz, poly(propylene azelate); PPPim, poly(propylene pimalate); PPGlu, poly(propylene glutarate); PPAd, poly(propylene adipate).

solubility and the low mean nanoparticle diameter size, enabling drug diffusion to take place easily.

Comparing the release rates of ropinirole $\mathrm{HCl}$ from the different aliphatic polyesters, it can be seen that the release rates are higher for PPAz and PPPim and lower for PPGlu and PPAd. Furthermore, it is also observed that release of ropinirole $\mathrm{HCl}$ from PPAz and PPPim reaches $100 \%$, while for PPGlu and PPAd it is $40 \%$ and $20 \%$, respectively. Taking into consideration the fact that the hydrolysis rate for the studied aliphatic polyesters at $37^{\circ} \mathrm{C}$ is less than $2.4 \%$ in the

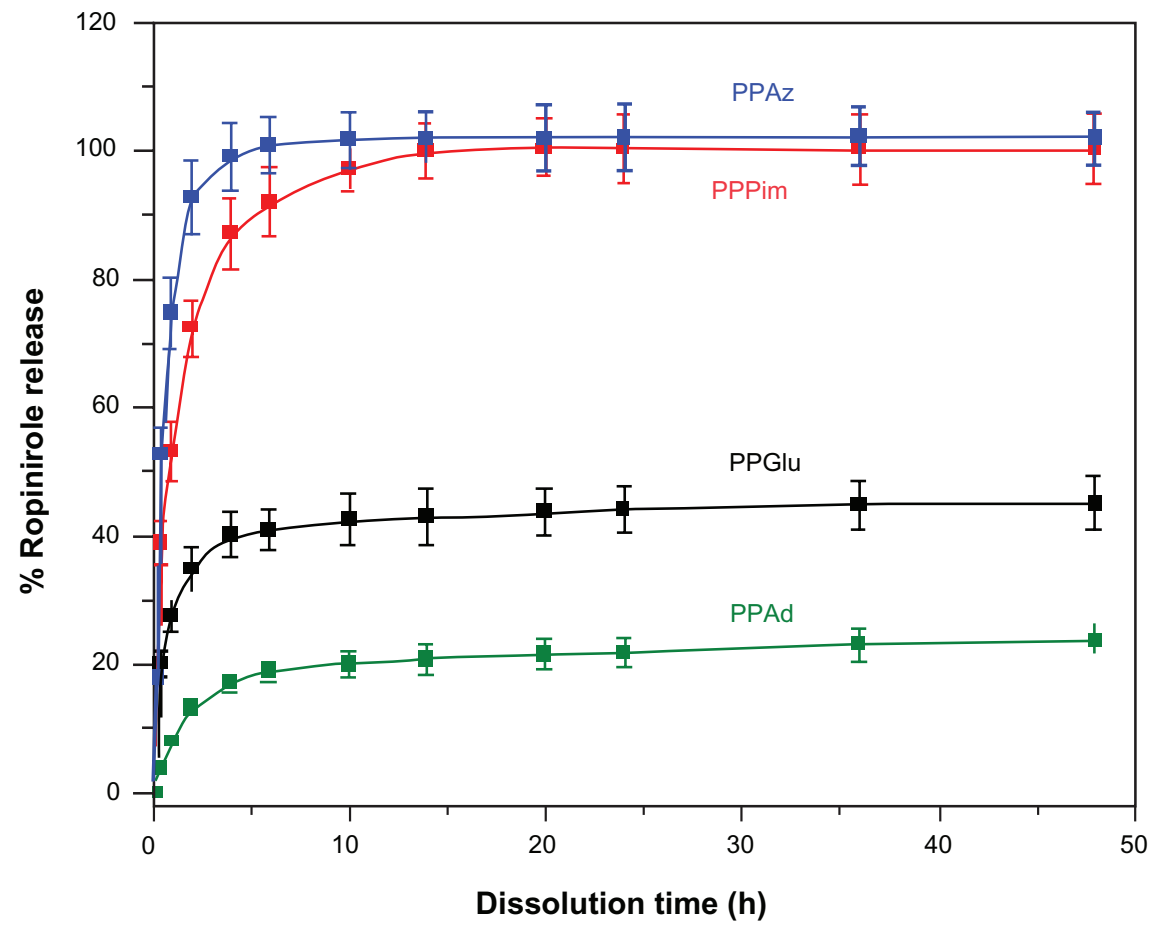

Figure 7 Release profiles for ropinirole $\mathrm{HCl}$ from polyester nanoparticles.

Abbreviations: PPAz, poly(propylene azelate); PPPim, poly(propylene pimalate); PPGlu, poly(propylene glutarate); PPAd, poly(propylene adipate). 
absence of enzymes,${ }^{35}$ it is believed that the drug release is not caused by erosion of the polymer matrices, since the degradation of these nanoparticles appears to be limited. It is our strong impression that the profile of ropinirole $\mathrm{HCl}$ release from nanoparticles is mainly due to diffusion. In this situation, the mean size of the nanoparticles could play a key role in the release profile, as reported above, and the release rate should be higher from nanoparticles of small size. If this was the main factor affecting drug release, then PPGlu should have the highest release rate because it has the lowest mean particle size, while PPAz should have the lowest release rate. However, from Figure 7, such a pattern of release behavior was not observed. Nevertheless, in our study, we cannot conclude that the effect of particle size is negligible. Furthermore, our data for particle size distribution (Figure 4) show relatively high polydispersity values, so it can be said that the actual sizes of the different batches are very close and overlapping may be expected. The zeta potential and possibly particle surface charge could also have a contributory effect. However, the types of polyesters used in this study were very hydrophobic materials with a negative zeta potential $(-15$ to -18$)$. This is due to the existence of hydroxyl and mainly carboxyl end groups in the prepared polyesters. Thus, it is to be expected that the nanoparticles we prepared would behave in a similar way.

A factor that might also play a key role in the release profile of ropinirole $\mathrm{HCl}$ is the polymer structure and, as a result, molecular weight, crystallinity, melting point, and glass transition temperature, which can affect the release of the drug from the polymer matrix to the dissolution medium. ${ }^{33}$ High crystallinity could lead to formation of a microchannel structure, and at the same time the large surface area of the polymer matrix could cause the drug to be released easily from the nanoparticles. ${ }^{36}$ However, in the present study, it was verified that, using polyesters with a similar melting point and glass transition temperature but different degrees of crystallinity, the release rate of ropinirole $\mathrm{HCl}$ is lower from polyesters with a higher degree of crystallinity. This could also be verified for PPAz nanoparticles, in which the polyester has a high molecular weight, melting point, and glass transition temperature, compared with the other polyesters. However, the release rate of ropinirole $\mathrm{HCl}$ is higher from all other nanoparticles. This is because PPAz has the lowest degree of crystallinity (29.5\%). Furthermore, PPAd, which has a low molecular weight and the lowest glass transition temperature, has the slowest drug release due to its high degree of crystallinity. This is in agreement with other studies reporting that high crystallinity of the matrix may have a negative influence on the release rate of a drug, because the lamellae may act as a barrier during drug diffusion. ${ }^{18,37,38}$ Bigger and more perfectly shaped crystalline lamellae should reduce overall release of the drug. This is probably the reason for higher release rates in the case of PPAz and PPPim, which show a low degree of crystallinity, ie, $29.5 \%$ and $42.6 \%$ respectively. In contrast, very low release rates were obtained for PPAd and PPGlu, which have a higher degree of crystallinity at $62.6 \%$ and $51.9 \%$, respectively. Diffusion of the active pharmaceutical ingredient through the amorphous matrix seems to be easier due to the higher mobility of the macromolecular chains of the polyester in the amorphous state, and thus easier penetration of water through them and, consequently, a faster release rate for ropinirole is obtained.

\section{Conclusion}

We prepared polyesters with high molecular weight, similar glass transition temperatures and melting points, but very different degrees of crystallinity, ranging from $29.8 \%$ to $67.5 \%$. All the polyesters demonstrated low cytotoxicity, so may be used as drug carriers for nanoencapsulation of a freely soluble drug like ropinirole $\mathrm{HCl}$. Drug loading, entrapment efficiency, and particle size of the prepared nanoparticles may be considered satisfactory, keeping in mind that a highly soluble drug was used, while in all cases the drug was encapsulated in an amorphous form. The rate of ropinirole $\mathrm{HCl}$ release was affected by drug solubility, as well as the degree of crystallinity of the polyesters used. Thus, the release rate is higher in polyesters having a low degree of crystallinity, due to higher macromolecular chain mobility.

\section{Acknowledgment}

This work was funded by the General Secretariat of Research and Technology of Greece in the framework of the PABET-NE project.

\section{Disclosure}

The authors have no conflicts of interest to report in this work.

\section{References}

1. Pillai O, Panchagnula R. Polymers in drug delivery. Curr Opin Chem Biol. 2001;5:447-451.

2. Lin YQ, You HB. Polymer architecture and drug delivery. Pharm Res. 2006;23:1-30.

3. Lakschmi SN, Cato TL. Biodegradable polymers as biomaterials. Prog Polym Sci. 2007;32:762-798.

4. Chasin M, Langer R, editors. Biodegradable Polymers as Drug Delivery Systems. New York, NY: Marcel Dekker Inc; 1990. 
5. Mohaned F, Van der Walle CF. Engineering biodegradable polyester particles with specific drug targeting and drug release properties. J Pharm Sci. 2008;97:71-87.

6. Moghimi SM, Hunter AC, Murray JC. Long-circulating and targetspecific nanoparticles: Theory to practice. Pharmacol Rev. 2001;53: 283-318.

7. Emerich D, Thanos CG. The pinpoint promise of nanoparticle-based drug delivery and molecular diagnosis. Biomol Eng. 2006;23: 171-184.

8. Des Rieux A, Fievez V, Garinot M, Schneider YJ, Preat V. Nanoparticles as potential oral delivery systems of proteins and vaccines: a mechanistic approach. J Control Release. 2006;116:1-27.

9. Vauthier C, Bouchemal K. Methods for the preparation and manufacture of polymeric nanoparticles. Pharm Res. 2009;26:1025-1058.

10. Rao JP, Geckeler KE. Polymer nanoparticles: preparation techniques and size control parameters. Prog Polym Sci. 2011;36:887-913.

11. Panyam J, Labhasetwar V. Biodegradable nanoparticles for drug and gene delivery to cells and tissue. Adv Drug Deliv Rev. 2003;55: 329-347.

12. Couvreur P, Vauthier C. Nanotechnology: intelligent design to treat complex disease. Pharm Res. 2006;23:1417-1450.

13. Torchilin VP. Micellar nanocarriers: pharmaceutical perspectives. Pharm Res. 2007;24:1-16.

14. Rawat M, Singh D, Saraf S. Nanotechcarriers: Promising vehicle for bioactive drugs. Biol Pharm Bull. 2006;29:1790-1798.

15. Ge H, Hu Y, Jiang, X, et al. Preparation, characterization, and drug release behaviors of drug nimodipine-loaded poly( $\varepsilon$-caprolactone)poly(ethylene oxide)-poly( $\varepsilon$-caprolactone) amphiphilic triblock copolymer micelles. J Pharm Sci. 2002;91:1463-1473.

16. Gref R, Minamitake Y, Peracchia MT, Trubetskoy V, Torchilin V, Langer R. Biodegradable long-circulating polymeric nanospheres. Science. 1994;263:1600-1603.

17. Shin IG, Kim SY, Lee YM, Cho CS, Sung YK. Methoxy poly(ethylene glycol)/E-caprolactone amphiphilic block copolymeric micelle containing indomethacin. I. Preparation and characterization. J Control Release. 1998;51:1-11.

18. Jeong JC, Lee J, Cho K. Effects of crystalline microstructure on drug release behavior of poly( $\varepsilon$-caprolactone) microspheres. $J$ Control Release. 2003;92:249-258.

19. Papageorgiou GZ, Bikiaris DN. Crystallization and melting behavior of three biodegradable poly(alkylene succinates). A comparative study. Polymer. 2005;46:12081-12092.

20. Bikiaris DN, Papageorgiou GZ, Giliopoulos DJ, Stergiou CA. Correlation between chemical and solid-state structures and enzymatic hydrolysis in novel biodegradable polyesters. The case of poly(propylene alkanedicarboxylate)s. Macromol Biosci. 2008;8:728-740.

21. Karavelidis V, Giliopoulos D, Karavas E, Bikiaris D. Nanoencapsulation of a water soluble drug in biocompatible polyesters. Effect of polyesters melting point and glass transition temperature on drug release behavior. Eur J Pharm Sci. 2010;41:636-643.

22. Lu XF, Hay JN. Isothermal crystallization kinetics and melting behaviour of poly(ethylene terephthalate). Polymer. 2001;42:9423-9431.

23. Bikiaris D, Karavelidis V, Karavas E. Effectiveness of various drug carriers in controlled release formulations of raloxifene $\mathrm{HCl}$ prepared by melt mixing. Curr Drug Deliv. 2009;6:425-436.
24. Bikiaris D, Karavelidis V, Karavas E. Novel biodegradable polyesters. synthesis and application as drug carriers for the preparation of raloxifene $\mathrm{HCl}$ loaded nanoparticles. Molecules. 2009;14:2410-2430.

25. Bikiaris DN, Papadimitriou S, Papageorgiou GZ, Kanaze FI, Georgarakis M. Nanoencapsulation of nimodipine in novel biocompatible poly(propylene-co-butylene succinate) aliphatic copolyesters for sustained release. J Nanomaterials. 2009; art. no. 716242.

26. Athanasiou KA, Niederauer GG, Agrawal CM. Sterilization, toxicity, biocompatibility and clinical applications of polylactic acid/polyglycolic acid copolymers. Biomaterials. 1996;17:93-102.

27. Niwa T, Takeuchi H, Hino T, Kunou N, Kawashima Y. Preparations of biodegradable nanospheres of water-soluble and insoluble drugs with D,L-lactide/glycolide copolymer by a novel spontaneous emulsification solvent diffusion method, and the drug release behavior. $J$ Control Release. 1993;25:89-98.

28. Khoee S, Hassanzadeh S, Goliaie B. Effects of hydrophobic drugpolyesteric core interactions on drug loading and release properties of poly(ethylene glycol)-polyester-poly(ethylene glycol) triblock coreshell nanoparticles. Nanotechnology. 2007;18: art. no. 175602.

29. Pinto Reis C, Neufeld RJ, Ribeiro AJ, Veiga F. Nanoencapsulation I. Methods for preparation of drug-loaded polymeric nanoparticles. Nanomedicine. 2006;2:8-21.

30. Mittal G, Sahana DK, Bhardwaj V, Ravi Kumar MN. Estradiol loaded PLGA nanoparticles for oral administration: Effect of polymer molecular weight and copolymer composition on release behavior in vitro and in vivo. J Control Release. 2007;119:77-78.

31. Papadimitriou S, Bikiaris D. Novel self-assembled core-shell nanoparticles based on crystalline amorphous moieties of aliphatic copolyesters for efficient controlled drug release. J Control Release. 2009;138: 177-184.

32. Zhang $\mathrm{L}, \mathrm{Hu} \mathrm{Y}$, Jiang $\mathrm{X}$, Yang $\mathrm{C}, \mathrm{Lu} \mathrm{W}$, Yang YH. Camptothecin derivative-loaded poly(caprolactone-co-lactide)-b-PEG-bpoly(caprolactone-co-lactide) nanoparticles and their biodistribution in mice. J Control Release. 2004;96:135-148.

33. Ge H, Hu Y, Yang S, Jiang X, Yang C. Preparation, characterization, and drug release behaviors of drug-loaded $\varepsilon$-caprolactone/L-lactide copolymer nanoparticles. J Appl Polym Sci. 2000;75:874-882.

34. Vassiliou AA, Papadimitriou SA, Bikiaris DN, Mattheolabakis G, Avgoustakis K. Facile synthesis of polyester-PEG triblock copolymers and preparation of amphiphilic nanoparticles as drug carriers. J Control Release. 2010;148:388-395.

35. Papageorgiou GZ, Bikiaris DN. Synthesis, cocrystallization, and enzymatic degradation of novel poly(butylene-co-propylene succinate) copolymers. Biomacromolecules. 2007;8:2437-2449.

36. Izumikawa S, Yoshioka S, Aso Y, Takeda, Y. Preparation of poly(1lactide) microspheres of different crystalline morphology and effect of crystalline morphology on drug release rate. J Control Release. 1991:15:133-140.

37. Miyajima M, Koshika A, Okada JI, Kusai A, Ikeda M. Factors influencing the diffusion-controlled release of papaverine from poly (1-lactic acid) matrix. J Control Release. 1998;56:85-94.

38. Miyajima M, Koshika A, Okada JI, Ikeda M. Effect of polymer/basic drug interactions on the two-stage diffusion-controlled release from a poly(-lactic acid) matrix. J Control Release. 1999;61:295-304.
International Journal of Nanomedicine

\section{Publish your work in this journal}

The International Journal of Nanomedicine is an international, peerreviewed journal focusing on the application of nanotechnology in diagnostics, therapeutics, and drug delivery systems throughout the biomedical field. This journal is indexed on PubMed Central, MedLine, CAS, SciSearch ${ }^{\circledR}$, Current Contents ${ }^{\circledR} /$ Clinical Medicine, Journal

\section{Dovepress}

Citation Reports/Science Edition, EMBase, Scopus and the Elsevier Bibliographic databases. The manuscript management system is completely online and includes a very quick and fair peer-review system, which is all easy to use. Visit http://www.dovepress.com/ testimonials.php to read real quotes from published authors. 